Original article

\title{
New system for predicting the outcome of secondary peritonitis
}

\author{
Nikolay V. Lebedev ${ }^{1}$, Sariya B. Agrba ${ }^{2}$, Vasily S. Popov ${ }^{1}$, Alexey E. Klimov ${ }^{1}$, Giorgy T. Svanadze ${ }^{1}$ \\ 1 Peoples' Friendship University of Russia (RUDN University), Moscow, Russia \\ 2 Burdenko National Medical Research Center for Neurosurgery, Moscow, Russia
}

Received 16 March 2021, Revised 24 May 2021, Accepted 28 July 2021

C 2021, Russian Open Medical Journal

\begin{abstract}
Despite improvements in the methods of diagnostics, surgical interventions and intensive care, the problem of treating patients with diffuse peritonitis remains relevant. Diffuse peritonitis is a major contributor to mortality in all urgent care settings and the second leading cause of sepsis in critically ill patients. At the same time, even in developed countries, the number of patients with peritonitis does not tend to decrease, and mortality rates remain high, reaching $90-93 \%$ with the development of abdominal sepsis and toxic shock syndrome. One of the ways to reduce mortality in peritonitis is the use of objective systems for prognosis of the peritonitis outcome, allowing to compare the results of patient treatment and to choose the optimal treatment tactics for each particular patient.

The objective - To develop a new system for predicting the outcome of secondary peritonitis (survival or death) focused on the criteria of abdominal sepsis and multiple organ dysfunction syndrome (associated or not associated with peritonitis), and to analyze its accuracy versus the most common comparable systems.

Material and Methods - Our study was based on analyzing the treatment outcomes in 352 patients with secondary diffuse peritonitis. On admission, sepsis was diagnosed in 15 (4.3\%), and toxic shock in 4 (1.1\%) patients. The main causes of death were purulent intoxication and/or sepsis (51 cases or $87.9 \%$ ), cancer intoxication ( 4 cases or $6.9 \%$ ), and acute cardiac failure (3 cases or $5.2 \%)$. We analyzed the effectiveness of several systems of predicting the peritonitis outcomes: the Mannheim's Peritoneal Index (MPI), World Society for Emergency Surgery Sepsis Severity Score (WSES SSS), Acute Physiology and Chronic Health Evaluation II (APACHE II) system, general Sequential Organ Failure Assessment Score (gSOFA), as well as the Peritonitis Prognosis System (PPS) developed by the authors. The probability of the effect of 40 clinical and laboratory parameters on the outcome of patients with secondary peritonitis was analyzed via using parametric and nonparametric methods of statistical analysis (Fisher's test, Mann-Whitney U test, Chi-squared test with Yates's continuity correction). The criteria were selected that had a predictive power for the lethal outcome $(p<0.05)$, and they were included in the PPS system. To compare the predictive value of the PPS, ROC analysis was conducted with construction of receiver operating characteristic curves for each analyzed system of predicting the peritonitis outcome. The STATISTICA 8 software was used for performing the statistical analysis.

Results - The following criteria were of greatest importance in predicting the lethal outcome: a patient's age, a presence of a malignant neoplasm, a nature of the exudate, the development of sepsis (toxic shock), as well as multiple organ dysfunction not associated with the developed peritonitis. PPS exhibited the greatest accuracy in terms of predicting mortality in patients with secondary diffuse peritonitis ( $A \cup C=0.942$ ) versus minimal in APACHE II (AUC=0.840).

Conclusion - APACHE II, MPI, WSES SSS and PPS can be considered reliable in terms of mortality prognosis in peritonitis patients. PPS has the greatest accuracy of predicting the mortality in patients with secondary diffuse peritonitis (94\%).
\end{abstract}

Keywords: Peritonitis, prognosis systems, abdominal sepsis, multiple organ dysfunction syndrome.

Cite as Lebedev NV, Agrba SB, Popov VS, Klimov AE, Svanadze GT. New system for predicting the outcome of secondary peritonitis. Russian Open Medical Journal 2021; 10: e0315.

Correspondence to Nikolay V. Lebedev. Email: nickl55@mail.ru.

\section{Introduction}

A preliminary assessment of the lethality outcome likelihood is used for selecting the rational treatment tactics, along with comparing the treatment outcomes and scientific research results.

Currently, there are several general clinical systems, along with specific schemes, for predicting the outcome of peritonitis, which more or less combine various factors for early prediction of treatment results, but none of such approaches, according to Tolonen M. et al. (2018), work satisfactorily [1].
These algorithms are useful for comparing large groups of patients, but each system has its own drawbacks, the main of which is that they all have limited value in the daily clinical decision-making process for each individual patient [2]

Perhaps this is due to the fact that the parameters of such systems do not take into account (or take into account only superficially) pathological changes in the abdominal cavity observed by the surgeon during the operation [3]. Nonetheless, at present, the following systems for predicting the outcome of peritonitis are widely used: Acute Physiology and Chronic Health 
Evaluation II (APACHE II) [4], Simplified Acute Physiology Score (SAPS II) [5], Sequential Organ Failure Assessment Score (SOFA) [6], Mannheim Peritoneal Index (MPI), and World Society for Emergency Surgery Sepsis Severity Score (WSES SSS) [7].

However, APACHE II is of little use in the choice of surgical tactics for treating a patient with peritonitis, since it does not take into account pathological changes in the abdominal cavity and the cause of peritonitis [8]. Besides, APACHE II is aimed at classifying patient groups rather than individual patients, making it difficult to apply to a particular patient [9].

Results of assessment via APACHE II are often reported in the literature as a score rather than a percentage of lethal outcomes. Given that APACHE II scores are presented to describe disease severity in a population, it could be difficult to accurately compare two incongruent patient populations because predicted mortality is based on indications at admission to the intensive care unit.

Use of any specific biochemical parameter as a prognostic indicator is not common [10], and virtually all studied biomarkers of sepsis to date have insufficient specificity due to the development of one or another degree of systemic inflammation in various categories of patients at intensive care units [11].

Comparison of the published data casts doubt on the reliability of the MPI prediction of the peritonitis outcome without taking into account its nature and cause. For example, Billing et al., (1994) considered the threshold value of the assessment at the level of $26 \mathrm{MPI}$ points. For this value, the average sensitivity of the test was $86 \%$ (54-98\%), its average specificity was $74 \%$ (58-97\%), and the accuracy of predicting lethal outcome was $83 \%$. Depending on MPI scores, average mortality of the patients varied: for a score of under 21 points, an average mortality was 2.3\%; for 21-29 points, it was 22.5\%; and for severity score of over 29 points, the mortality was $59.1 \%$ [12]. However, when comparing the results of the mentioned study with the data of nine independent studies of mortality in peritonitis, depending on the MPI score, it could be seen that with the same assessment parameter values, deaths occur with different probabilities, and the difference reaches $50 \%$ [13].

It is possible that the differences in the mortality rate, depending on the MPI score [14], were caused by different distribution of patients in terms of peritonitis. Unfortunately, in their publications, the authors, as a rule, did not indicate clearly how the patients were distributed by the main diagnosis. According to N.V. Lebedev et al (2017), MPI can be used to evaluate treatment outcomes and research results only in patients with the same cause of peritonitis [15].

Many studies confirmed the accuracy of the WSES SSS in predicting mortality in patients with peritonitis $[16,17,18]$

At the same time, some studies on the comparative characterization of systems for predicting the outcome of peritonitis raise doubts about the accuracy of WSES SSS. For example, studies by Tolonen M. et. al (2019) showed that SOFA score $\geq 2(78.4 \%)$ had the highest identification rates, followed by WSES SSS $\geq 8$ (73.1\%), SOFA $\geq 3$ (75.2\%), and APACHE $\| \geq 14$ $(68.8 \%)$. The most accurate prediction took place at SOFA score $\geq 2$ with $78.4 \%$ identification rates, followed by SOFA score $\geq 3$ (with $75.2 \%$ ), WSES SSS $\geq 8$ (with 73.1\%), and APACHE II $\geq 14$ (with $68.8 \%$ identification rates) [3]. According to Mohan R. et al. (2019), MPI is superior to WSES in predicting mortality in patients with peritonitis [19].
The PIPAS system (Physiological Indicators for Prognosis in Abdominal Sepsis) was proposed in 2019 [20]. The creators of this system believed that the PIPAS indicator had a very good ability to determine the likelihood of a lethal outcome.

However, the validity of PIPAS should be tested in future large prospective series before it could be recommended for use in clinical practice. A potential limitation of using PIPAS could possibly be a large proportion of patients with acute appendicitis included in the study (42.1\%) [21], as well as the inclusion of patients with severe pancreatitis, which has its own pathophysiological characteristics. Besides, this system does not take into account the intra-abdominal changes in peritonitis and, as a result, is not promising for choosing the surgical tactics. Probably, the PIPAS system is analogous to the general clinical system APACHE II or SAPS II.

The severity of a peritonitis patient condition is associated with a number of clinical and pathophysiological factors. The most important risk factors of a lethal outcome are: the nature of the abdominal lesion and the presence of sepsis with associated organ lesions $[2,22,23]$. At the same time, the presence or absence of abdominal sepsis is of particular importance for the outcome of peritonitis [24].

Other important factors include the physiological reserve of a patient, comorbidities, the state of immune system, and anatomical abnormalities caused by the disease.

The objective. To develop a novel system for predicting the outcome of secondary peritonitis (survival or death) focused on the criteria of abdominal sepsis and multiple organ dysfunction syndrome (MODS), associated or not associated with peritonitis, and to analyze its accuracy against the most common comparable systems.

\section{Material and Methods}

\section{Study design}

Our study was based on an analysis of the treatment results of 353 patients with secondary diffuse peritonitis. Patients with pancreatic necrosis and impaired mesenteric circulation were excluded from the study due to the peculiarities of pathogenesis and difficulties in predicting the further development of the disease. The clinical characteristics of study subjects are shown in Table 1.

Table 1. Clinical characteristics of patients, $n=353$

\begin{tabular}{lc}
\hline Parameter & Value \\
\hline Average age, years & $55.8 \pm 9.1$ \\
Men/women ratio & $1.2: 1.0(190: 163)$ \\
\hline$-y$ & Characteristics of peritonitis sensu V.S. Savelyev \\
\hline Diffuse & $336(95.5 \%)$ \\
Generalized & $16(4.5 \%)$ \\
\hline \multicolumn{1}{c}{ The most widespread causes of peritonitis, $\mathrm{n}=353$} \\
Acute destructive appendicitis & $122(34.6 \%)$ \\
Perforations of gastric and duodenal ulcers & $85(24.1 \%)$ \\
Colon perforation of various origins & $58(16.4 \%)$ \\
Acute destructive cholecystitis & $36(10.2 \%)$ \\
- & \\
& \\
Purulent intoxication and/or sepsis & $51(87.9 \%)$ \\
Cancer intoxication & $4(6.9 \%)$ \\
Acute cardiac failure & $3(5.2 \%)$ \\
Total lethality & $16.7 \%$ \\
\hline
\end{tabular}


Table 2. Impact of the criteria included in the PPS system on the prognosis

\section{Criteria}

Age $>80$ years

Concomitant cancer

Purulent exudate

Fecal exudate

gSOFA

Sepsis

Toxic shock

\section{Table 3. Peritonitis Prognosis System (PPS)}

\begin{tabular}{lc}
\hline Criteria & Score, points \\
\hline Age, years & 0 \\
under 60 & 3 \\
$61-70$ & 4 \\
$71-80$ & 5 \\
over 80 & 5 \\
\hline Presence of a malignant neoplasm & 3 \\
Exudate & 5 \\
Purulent & 5 \\
Fecal & 5 \\
Systemic inflammatory response & 7 \\
Sepsis & 4 \\
Toxic shock & \\
Organ failure not associated with peritonitis (comorbidity) & 4 \\
\hline
\end{tabular}

Table 4. The probability of death vs. the score sensu Peritonitis Prognosis System

\begin{tabular}{lccc}
\hline PPS score & Patient number & Died & Lethality (\%) \\
\hline $0-3$ & 208 & 1 & 0.48 \\
$4-6$ & 59 & 9 & 15.25 \\
$7-10$ & 51 & 21 & 41.20 \\
$11-14$ & 28 & 21 & 75.00 \\
15 and more & 7 & 7 & 100.00 \\
Total: & 353 & 59 & 16.70 \\
\hline
\end{tabular}

Table 5. Indicators of ROC analysis for compared peritonitis outcome prognosis systems

\begin{tabular}{lccccc}
\hline $\begin{array}{l}\text { Peritonitis } \\
\text { prognosis systems }\end{array}$ & $\begin{array}{c}\text { Area under the } \\
\text { curve }(A U C)\end{array}$ & $\begin{array}{c}\text { Sensitivity } \\
\text { (Se) }\end{array}$ & $\begin{array}{c}\text { Specificity } \\
\text { (Sp) }\end{array}$ & $\begin{array}{c}\text { Accuracy } \\
\text { (ACC) }\end{array}$ & $\begin{array}{c}P \text { values } \\
\text { PPS }\end{array}$ \\
\hline P & 0.942 & 92 & 98 & 98 & 0.003 \\
MPI & 0.908 & 30 & 98 & 93 & 0.006 \\
WSES SSS & 0.881 & 25 & 98 & 94 & 0.041 \\
APACHE II & 0.840 & 24 & 96 & 92 & 0.037 \\
\hline
\end{tabular}

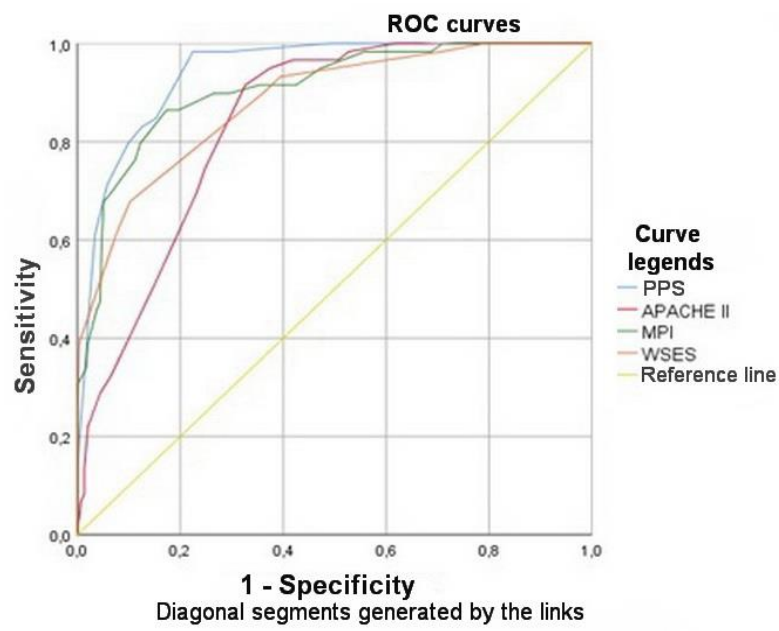

Figure 1. ROC curves of sensitivity and specificity for PPS, APACHE II, WSES SSS, and MPI systems in predicting peritonitis-caused mortality.
We analyzed the sensitivity and specificity of the Mannheim Peritoneal Index (MPI) [25], the Sepsis Severity Assessment of the World Society of Emergency Surgery (WSES SSS) for patients with complicated intra-abdominal infections (CIAI: APACHE-II systems) [26], gSOFA scores, as well as the Peritonitis Prognosis System (PPS) developed by the authors of this article.

Sensu the recommendations for sepsis-III, sepsis is understood as a sharp change of two or more points in the assessment of general Sequential Organ Failure Assessment (gSOFA); whereas toxic shock is characterized as sepsis with persistent hypotension requiring vasopressors to maintain an average arterial pressure $\geq$ $65 \mathrm{~mm} \mathrm{Hg}$ and persistent serum lactate content of $>2 \mathrm{mmol} / \mathrm{L}$ despite the adequate volume resuscitation [4].

\section{Data processing}

Statistical analysis was performed using the STATISTICA 8 software. A p-value less than 0.05 implied statistical significance, and the values of the probability of a predicted outcome (patient death) were given taking into account the $95 \%$ confidence interval [27].

To determine the statistical significance of the criteria effect on the outcome, we analyzed 40 clinical and laboratory parameters that could contribute to the outcome of peritonitis, using parametric and nonparametric methods of statistical analysis (Appendix 1). The principle of constructing the scale was based on the selection of parameters (indicated in Table 2) that were statistically significantly associated with death in patients with secondary peritonitis.

To compare the predictive value of such systems as MPI, APACHE II, WSES SSS and PPS, ROC (receiver operating characteristic) analysis was used with the construction of ROC curves for each system. The statistical difference in the predictive values of those systems was analyzed by comparing the area under the curve of each system. The positive predictive value (PPV) of an outcome, hereinafter referred to as predictive value, was also calculated: it is the proportion (probability) of unfavorable outcomes (death) among all patients with a high risk of death. The described indicators are relative values and are expressed as percentages.

\section{Results}

We revealed the prognostic factors significantly affecting $(p<0.05)$ the outcome of secondary peritonitis (Table 1$)$. As can be seen, the criteria of a patient age, the presence of a malignant neoplasm, a nature of the exudate, sepsis (toxic shock), as well as organ failure (gSOFA) not associated with the development of peritonitis were of the greatest importance in predicting the lethal outcome. These criteria, presented in Table 2, were statistically significantly associated with the lethal outcome of peritonitis $(p<0.05)$.

Based on the obtained data, we came up with a system for predicting peritonitis (PPS) (Table 3). The scoring system was developed taking into account the clinically significant, in our opinion, influence on the ongoing process of secondary peritonitis, as well as considering the level of their statistical significance. Weights (points) for each criterium were selected empirically.

The likelihood of a peritonitis outcome (ranks), depending on the number of points sensu PPS criteria, is presented in Table 4. 
To compare the predictive value of PPS versus the most widely used systems for predicting the outcome of peritonitis (MPI, APACHE II, WSES SSS), ROC analysis was used with the construction of ROC curves for each system. Statistical indicators and curves obtained as a result of the performed ROC analysis are shown in Table 5 and Figure 1.

The performed analysis suggested that PPS was the most sensitive of all tested systems $(\mathrm{Se}=92 \%)$. The greater sensitivity could be explained by a large number of true positive outcomes, that is, by a large number of deceased patients, for whom the mortality index exceeded the threshold level of $50 \%$. Statistically significant differences in predictive values were obtained for PPS, WSES SSS, and APACHE II systems ( $p<0.05$, Fisher's F Test). The MPI system exhibited statistically similar results in terms of assessing mortality in patients with secondary peritonitis in this sample of patients. The predictive value was calculated according to the criteria of the high risk of a patient death, assuming the threshold values of 26 points for MPI; 11 points for PPS; $\geq 7$ points for WSES SSS; and $\geq 14$ points for APACHE II. Hence, the predictive values were $38.2 \%$ for MPI, $83.3 \%$ for PPS, $74.3 \%$, for WSES SSS, and $50.9 \%$ for APACHE II. Thus, the likelihood of unfavorable outcomes among all patients at high risk of death was determined most accurately by PPS and WSES SSS.

The obtained results can be explained by the inclusion of the criteria related to mortality in the group of patients with secondary peritonitis in a thanatogenetic and pathophysiologic manner - specifically, the criteria directly leading to the development of abdominal infection, e.g., the purulent and fecal character of the exudate, sequential organ failure, sepsis and, as a consequence, cerebral edema, brain herniation syndrome and clinical death of a patient. As a result of the conducted analysis, the most accurate system, in terms of predicting mortality in a patient with secondary diffuse peritonitis, was the PPS. This was also confirmed by the AUC parameter (area under the ROC curve), had maximum values for PPS (0.942) and the minimum values for APACHE II (0.840). Such high predictive values of PPS and MPI could be explained by high sensitivities of these prediction systems specifically for the peritonitis. WE established that WSES SSS was less specific, since it was designed for urgent surgical diseases, without taking into account the peculiarities of the secondary peritonitis course. The general clinical APACHE II system was originally developed to assess the severity of the condition of intensive care patients and therefore was less focused specifically on peritonitis. For this reason, it demonstrated low specificity.

\section{Discussion}

Diffuse peritonitis is a major contributor to non-trauma mortality in all urgent care settings and the second leading cause of sepsis in critically ill patients [28-30]. Moreover, even in developed countries, the number of patients with peritonitis does not tend to decline, and their mortality rates remain high [31]. Mortality in diffuse peritonitis varies from 26 to $60 \%$, reaching a level of $90-93 \%$ with the development of abdominal sepsis and toxic shock syndrome [32, 33].

The prognosis of peritonitis outcome depends on a combination of several factors: the degree of severity of a patient state, resource capabilities of a patient, specific factors of the disease, and diagnostic and therapeutic interventions. The distribution of patients among different risk groups helps predicting the outcome of the illness, selecting patients for intensive care, and identifying the surgical risk, thereby contributing to the choice of surgical treatment tactics - for example, the semi-open method, programmed relaparotomy or, in exceptional cases, damage control tactics.

One of the leading risk factors for peritonitis is preoperative sepsis with associated organ dysfunctions and toxic shock [34]. Other factors include a patient's adaptive characteristics, concomitant diseases, especially those entailing organ failure, immunosuppression, and anatomical disorders associated with the disease $[22,33]$.

The results of our study suggested that abdominal sepsis and multiple organ dysfunction syndrome (associated or not associated with peritonitis) were the most important risk factors of a patient's death caused by the peritonitis.

Of course, when choosing the tactics of treating a patient with peritonitis, it is necessary to take into account the degree of pathological changes in the abdominal cavity (fibrin deposition, the state of intestinal loops, and degree of their dilatation). However, the inclusion of these parameters or systems in prediction of the peritonitis outcome is not frequent. The good example supporting this statement is represented by inclusion of the Abdominal Cavity Index [6] in the system for predicting the outcome of peritonitis: it is very problematic to use in practice, especially because the contribution of each symptom (except for the nature of exudate) is quite small.

A low score of systems for predicting the outcome of peritonitis does not guarantee a favorable outcome of the disease. Technical and tactical errors in the treatment of patients may lead to severe complications. For example, in the analyzed group of patients, a 53-year-old woman (on admission, APACHE II of 6 points, MPI of 22 points, PPS of 3 points) with a perforated duodenal ulcer has developed suture failure and progression of peritonitis on the second day after suturing the ulcer. The patient underwent repeated suturing of the ulcerative defect, drainage of the abdominal cavity, and management according to the program of sanitation relaparotomies (4 relaparotomies in total). The death occurred due to the onset of tertiary peritonitis and purulent intoxication.

We would also like to admit that our results are based on the treatment outcomes at just one clinic; hence they require further independent external verification.

\section{Conclusions}

1. APACHE II, MPI, WSES SSS and PPS systems can be considered reliable in terms of mortality prognosis in patients with peritonitis and could be used as alternatives.

2. The greatest accuracy (94\%) and predictive value $(83.3 \%)$ in assessing the likelihood of a lethal outcome in patients with secondary diffuse peritonitis was established for the Peritonitis Prognosis System. The Mannheim's Peritoneal Index also exhibited high predictive accuracy (over $90 \%$ ).

\section{Funding}

The study was carried out in accordance with the research plan of the Medical Institute at the Peoples' Friendship University of Russia.

\section{Conflict of interest}

The authors declare that they have no conflicts of interest. 


\section{Ethical approval}

The study was approved by the Ethics Committee at the Peoples' Friendship University of Russia. Ethical endorsement applied to all procedures performed in research involving humans, to the ethical standards of the institutional and national research committee, and to the 1964 Declaration of Helsinki and its later amendments or comparable ethical standards. For this type of study, formal informed consent was not required.

\section{References}

1. Tolonen M, Coccolini F, Ansaloni L, Sartelli M, Roberts DJ, McKee JL, et al. Getting the invite list right: a discussion of sepsis severity scoring systems in severe complicated intra-abdominal sepsis and randomized trial inclusion criteria. World J Emerg surg 2018; 13: 17. https://doi.org/10.1186/s13017-018-0177-2.

2. Tolonen $M$, Sallinen $V$, Mentula $P$, Leppaniemi A. Preoperative prognostic factors for severe diffuse secondary peritonitis: a retrospective study. Langenbecks Arch Surg 2016; 401(5): 611-617. https://doi.org/10.1007/s00423-016-1454-8.

3. Tolonen $M$, Sallinen V, Leppäniemi A, Bäcklund M, Mentula P. The role of the intra-abdominal view in complicated intra-abdominal infections. World J Emerg Surg 2019; 14: 15. https://doi.org/10.1186/s13017-0190232-7.

4. Knaus WA, Draper EA, Wagner DP. APACHE II: A severity of disease classification system. Crit Care Med 1985; 13(10): 818-829. https://doi.org/10.1097/00003246-198510000-00009.

5. Le Gall JR, Lemeshow S, Saulnier F. A new Simplified Acute Physiology Score (SAPS II) based on a European/North American multicenter study. JAMA 270(24): 2957-2963 https://doi.org/10.1001/jama.270.24.2957.

6. Vincent JL, de Mendonça A, Cantraine F, Moreno R, Takala J, Suter PM, et al. Use of the SOFA score to assess the incidence of organ dysfunction/failure in intensive care units: Results of a multicenter, prospective study. Working group on "sepsis-related problems" of the European Society of Intensive Care Medicine. Crit Care Med 1998; 26(11): 1793-1800 https://doi.org/10.1097/00003246-19981100000016.

7. Abdel-Kader S, Sartelli M, Abu-Zidan FM. Complicated intra-abdominal infections: A prospective validation study of the WSES Sepsis Severity Score. Singapore Med J 2019; 60(6): 317-321. https://doi.org/10.11622/smedj.2018120.

8. Knaus WA, Zimmerman JE, Wagner DP, Draper EA, Lawrence DE. APACHE - acute physiology and chronic health evaluation: A physiologically based classification system. Crit Care Med 1981; 9(8): 591-597 https://doi.org/10.1097/00003246-198108000-00008.

9. Parsons PE, Wiener-Kronish JP. Critical Care Secrets. 4th Ed, Mosby. 2007; 550 p. https://doi.org/10.1016/B978-1-4160-3206-9.X1000-2

10. Aliev SA, Aliev ES. Abdominal sepsis: The state of the problem, integral system for assessing the severity of sepsis and criteria for predicting the outcome. Grekov's Bulletin of Surgery 2018; 177(5): 108-112. Rusian. https://doi.org/10.24884/0042-4625-2018-177-5-108-112.

11. Saveliev VS, Gelfand BR, Filimonov MI, eds. Peritonitis. A Practical Guide. Moscow, Russia: Littera, 2006. 208 p. Russian.

12. Billing A, Fröhlich D, Schildberg FW. Prediction of outcome using the Mannheim peritonitis index in 2003 patients. Peritonitis Study Group. $\mathrm{Br} \quad J \quad$ Surg 1994; 81(2): 209-213. https://doi.org/10.1002/bjs.1800810217.

13. Lebedev NV, Klimov AE, Agrba SB, Gaidukevich EK. Combined forecasting system of peritonitis outcome. Pirogov Russian Journal of $\begin{array}{llll}\text { Surgery 2017; } & \text { (9): } & \text { 337. }\end{array}$ https://doi.org/10.17116/hirurgia2017933-37.

14. Ouf TI, Jumuah WAA, Mahmoud MA, Abdelbaset RI. Mortality rate in patients with secondary peritonitis in Ain Shams University Hospitals as regard Mannheim Peritonitis Index (MPI) score. QJM: An
International Journal of Medicine 2020; 113(Suppl 1): hcaa050.073 https://doi.org/10.1093/qjmed/hcaa050.073.

15. Irpatgire RN, Badane A. Efficacy of Mannheim peritonitis index (MPI) score in patients with perforation peritonitis. Int J Surg Sci 2019; 3(4) 457-459 https://doi.org/10.33545/surgery.2019.v3.i4h.285.

16. Payá-Llorente C, Martínez-López E, Sebastián-Tomás JC, SantarrufinaMartínez S, de'Angelis N, Martínez-Pérez A. The impact of age and comorbidity on the postoperative outcomes after emergency surgical management of complicated intra-abdominal infections. Sci Rep 2020; 10(1): 1631. https://doi.org/10.1038/s41598-020-58453-1.

17. Barie PS, Hydo $L$, Eachempati SR. Longitudinal outcomes of intraabdominal infection complicated by critical illness. Surg Infect (Larchmt) 2004; 5(4): 365-373. https://doi.org/10.1089/sur.2004.5.365.

18. Mwenda KI, Ojuka D, Awory M. Utility of World Society of Emergency Surgery Sepsis Severity Score in predicting outcomes of intraabdominal infections. Ann Afr Surg 2020; 17(2): 65-68 https://doi.org/10.4314/aas.v17i2.5.

19. Geethapriya S, Kannan R. Possum scoring in hollow viscus perforation. IOSR Journal of Dental and Medical Sciences 2017; 16(2): 102-108 https://doi.org/10.9790/0853-160206102108.

20. Raimondo S, Sartelli M, Coccolini F, Fugazzola P, Bova R, Tomasoni M, et al. Which prognostic score for abdominal sepsis? Analysis of final results of PIPAS (Physiological Indicators for Prognosis in Abdominal Sepsis) study in a single center. Journal of Peritoneum (and other serosal surfaces) $2018 ; \quad 3$ : 27-34. https://doi.org/10.4081/joper.2018.106.

21. Gomes CA, Sartelli M, Podda M, Di Saverio S, Coccolini F, SegoviaLohse HA, et al. Laparoscopic versus open approach for diffuse peritonitis from appendicitis etiology: A subgroup analysis from the Physiological Parameters for Prognosis in Abdominal Sepsis (PIPAS) study. Updates Surg 2020; 72(1): 185-191. https://doi.org/10.1007/s13304-020-00711-y.

22. Sartelli M, Catena F, Abu-Zidan FM, Ansaloni L, Biffl WL, Boermeester $\mathrm{MA}$, et al. Management of intra-abdominal infections: Recommendations by the WSES 2016 consensus conference. World J Emerg Surg 2017; 12: 22. https://doi.org/10.1186/s13017-017-0132-7.

23. Singer M, Deutschman CS, Seymour CW, Shankar-Hari M, Annane D, Bauer $\mathrm{M}$, et al. The Third International Consensus Definitions for Sepsis and Septic Shock (Sepsis-3). JAMA 2016; 315(8): 801-810. https://doi.org/10.1001/jama.2016.0287.

24. Kirkpatrick AW, Coccolini F, Ansaloni L, Roberts DJ, Tolonen M, McKee $\mathrm{JL}$, et al. Closed Or Open after Laparotomy (COOL) after Source Control for Severe Complicated Intra-Abdominal Sepsis Investigators. Closed Or Open after Source Control Laparotomy for Severe Complicated Intra-Abdominal Sepsis (the COOL trial): Study protocol for a randomized controlled trial. World J Emerg Surg 2018; 13: 26. https://doi.org/10.1186/s13017-018-0183-4.

25. Savelyev VS, Gelfand BR, eds. Abdominal surgical infection. National recommendations. Moscow, Russia: Borges. 2011; 98 p. Russian. https://www.elibrary.ru/item.asp?id=26054509.

26. Wacha $H$, Linder MM, Feldmann $U$, Wesch $G$, Steifensand RA, Gundlach E. Der Mannheimer Peritonitis-Index - ein Instrument zur intraoperativen Prognose der Peritonitis. In: Wacha H. Peritonitis. Grundsätzliches zur Therapie. Berlin, Heidelberg: Springer. 1987: 73100. German. https://doi.org/10.1007/978-3-642-73008-5 6.

27. Robin X, Turck N, Hainard A, Tiberti N, Lisacek F, Sanchez JC, et al. pROC: An open-source package for $\mathrm{R}$ and $\mathrm{S}+$ to analyze and compare ROC curves. BMC Bioinformatics 2011; 12: 77. https://doi.org/10.1186/1471-2105-12-77.

28. Belokonev VI, Izmailov TP. Diagnostics and treatment of gastrointestinal tract fistulas. Samara, Russia: Perspektiva. 2005; 238 p. Russian. https://www.elibrary.ru/item.asp?id=19532664.

29. Chichom-Mefire A, Fon TA, Ngowe-Ngowe M. Which cause of diffuse peritonitis is the deadliest in the tropics? A retrospective analysis of 
305 cases from the south-west region of Cameroon. World J Emerg Surg 2016; 11: 14. https://doi.org/10.1186/s13017-016-0070-9.

30. Bassetti M, Eckmann C, Giacobbe DR, Sartelli M, Montravers P. Postoperative abdominal infections: Epidemiology, operational definitions, and outcomes. Intensive Care Med 2019; 46(2): 163-172. https://doi.org/10.1007/s00134-019-05841-5.

31. Müller V, Koplin G, Pratschke J, Raue W. The treatment of acute secondary peritonitis: A retrospective analysis of the use of continuous negative pressure therapy. Med Klin Intensivmed Notfmed 2018; 113(4): 299-304. German. https://doi.org/10.1007/s00063-017-03096.

32. Green S, Kong VY, Clarke DL, Sartorius B, Odendaal J, Bruce JL, et al. The spectrum and outcome of surgical sepsis in Pietermaritzburg, South Africa. S Afr Med J 2017; 107(2): 134-136. https://doi.org/10.7196/samj.2017.v107i2.11339.

33. Bensman VM, Savchenko YuP, Sahakyan EA, Malyshko VV. Peritoneum viscera-parietal adhesive disease and laparotomy wound healing with peritonitis. Siberian Medical Review 2019; (5): 72-79. Russian. https://doi.org/10.20333/2500136-2019-5-72-79.

34. Pieracci FM, Barie PS. Management of severe sepsis of abdominal origin. Scand J Surg 2007; 96(3): 184-196 https://doi.org/10.1177/145749690709600302.

Authors:

Nikolay V. Lebedev - MD, DSc, Professor, Department of Faculty Surgery, Peoples' Friendship University of Russia, Moscow, Russia. https://orcid.org/0000-0002-7745-1390.

Sariya B. Agrba - clinical resident specializing in neurosurgery, Burdenko National Medical Research Center for Neurosurgery, Moscow, Russia. https://orcid.org/0000-0003-1668-6409.

Vasily S. Popov - MD, PhD, Instructor, Department of Faculty Surgery, Peoples' Friendship University of Russia, Moscow, Russia. https://orcid.org/0000-0001-7415-6794.

Alexey E. Klimov - MD, DSc, Professor, Chair of the Department of Faculty Surgery, Peoples' Friendship University of Russia, Moscow, Russia. https://orcid.org/0000-0002-0001-8003.

Giorgy T. Svanadze - Instructor, Department of Faculty Surgery, Peoples' Friendship University of Russia, Moscow, Russia. https://orcid.org/00000002-5420-475X. 
Appendix 1. Studied clinical and laboratory indicators of the lethality risk in a patient with peritonitis

Gender, age, time since the onset of the disease, the source of peritonitis, the presence of a malignancy, the presence of organ failure (associated or not associated with peritonitis), localized or diffuse or generalized peritonitis, stomach rumble, the nature of the exudate in the abdominal cavity and its volume, the presence or absence of sepsis, the presence or absence of toxic shock, leukocytes in the blood, hemoglobin, hematocrit, red blood cells, body temperature, average arterial pressure, systolic arterial pressure, heart rate, respiratory rate, oxygenation (A-aDO2 or $\mathrm{PaO2}$ ), $\mathrm{mm} \mathrm{Hg}$, sodium, potassium, serum creatinine, Glasgow Coma Scale (eye opening, motor response, speech), urea nitrogen, serum albumin, glucose, bilirubin, assessment of the acid-base status $(\mathrm{pH} / \mathrm{pCO} 2)$, urine erythrocytes and protein, procalcitonin, C-reactive protein, presepsin, central venous pressure, capillaroscopy data, results of ultrasound examination of the abdominal and pleural cavities. 